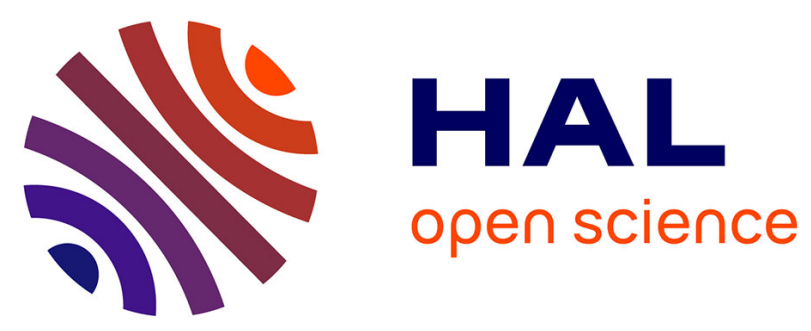

\title{
Risk factors for death and survival in paraneoplastic pemphigus associated with hematologic malignancies in adults
}

Elise Ouedraogo, Jeremy Gottlieb, Adèle de Masson, Clémence Lepelletier, Marie Jachiet, Camille Salle de Chou, Lionel Galicier, Martine Bagot, Sylvie

Chevret, Jean-David Bouaziz

\section{To cite this version:}

Elise Ouedraogo, Jeremy Gottlieb, Adèle de Masson, Clémence Lepelletier, Marie Jachiet, et al.. Risk factors for death and survival in paraneoplastic pemphigus associated with hematologic malignancies in adults. Journal of The American Academy of Dermatology, 2019, 80, pp.1544 - 1549. 10.1016/j.jaad.2018.03.043 . hal-03480667

\section{HAL Id: hal-03480667 https://hal.science/hal-03480667}

Submitted on 20 Dec 2021

HAL is a multi-disciplinary open access archive for the deposit and dissemination of scientific research documents, whether they are published or not. The documents may come from teaching and research institutions in France or abroad, or from public or private research centers.
L'archive ouverte pluridisciplinaire HAL, est destinée au dépôt et à la diffusion de documents scientifiques de niveau recherche, publiés ou non, émanant des établissements d'enseignement et de recherche français ou étrangers, des laboratoires publics ou privés.

\section{(ㄷ)(1) $\$$}

Distributed under a Creative Commons Attribution - NonCommerciall 4.0 International 
1 Risk factors for death and survival in paraneoplastic pemphigus associated with

\section{2 haematological malignancies in adults}

3 Elise Ouedraogo, $\mathrm{MD}^{1}$, Jeremy Gottlieb, $\mathrm{MD}^{1}$, Adèle de Masson, $\mathrm{MD}, \mathrm{PhD}^{1,2}$, Clémence 4 Lepelletier, $\mathrm{MD}^{1,2}$, Marie Jachiet, $\mathrm{MD}^{1,2}$, Camille Salle de Chou, $\mathrm{MD}^{1,2}$, Lionel Galicier, $\mathrm{MD}^{2,3}$, 5 M. Bagot, MD, $\mathrm{PhD}^{1,2}$, S. Chevret, MD, $\mathrm{PhD}^{2,4^{*}}$, J.D. Bouaziz, MD, $\mathrm{PhD}^{1,2^{*}}$

$6 \quad$ *Have equally contributed and share last author seniorship

7 Assistance Publique- Hôpitaux de Paris, Département de Dermatologie, Hôpital Saint-Louis, 8 Paris, France

$9 \quad{ }^{2}$ Université Paris-Diderot, Sorbonne Paris Cité

${ }^{3}$ Assistance Publique-Hôpitaux de Paris, Service d'Immuno-Hématologie, Hôpital Saint-

Louis, Paris, France

${ }^{4}$ Assistance Publique-Hôpitaux de Paris, Service de Santé publique, Hôpital Saint-Louis, Paris, France

\section{Corresponding author:}

Paris Cité, Hôpital Saint Louis, 1 avenue Claude Vellefaux, 75475, Paris, France.

17 Fax number: + 33142494620 .

email: jean-david.bouaziz@sls.aphp.fr

Running title: Paraneoplastic pemphigus in haematological malignancy

Keywords: pemphigus, lymphoma, Castleman's disease, bullous pemphigoid

Word counts: Abstract: 160 words; capsule summary: 57 words; manuscript: 1057 words; 11 references; 7 Tables and 2 Figures, 1 supplemental material.

23 Funding sources: This article has no funding source.

24 Conflicts of interest: The authors have no conflicts of interest to declare. 


\section{Capsule summary}

27 - Paraneoplastic pemphigus (PNP) may be associated with hematological malignancies (HM).

$28 \cdot$ HM associated with PNP are mostly non-Hodgkin lymphoma, chronic lymphocytic leukemia

29 and Castleman disease.

30 - The survival rate of PNP associated with HM is low. Toxic epidermal necrolysis-like PNP

31 and PNP associated with bronchiolitis obliterans have an especially poor prognosis. 


\section{Abstract}

33 Background Paraneoplastic pemphigus (PNP) occurs more often in patients with 34 hematological malignancies (HM) than in solid cancer patients. Lung bronchiolitis obliterans 35 (BO) is a severe complication of PNP.

36 Objective To precise clinical and biological features in HM-associated PNP and identify 37 factors associated with mortality and survival.

38 Methods Systematic review of previously described cases of PNP associated with HM.

39 Results One hundred forty-four patients were included. HM were non-Hodgkin lymphoma 40 (52.78\%), chronic lymphocytic leukemia (22.92\%), Castleman disease (18.60\%) and "other 41 underlying hematologic malignancy" (5.70\%). The mortality rate was 57\%; most deaths 42 occurred within the first year of PNP diagnosis. Multivariate analysis showed that: i/ presence 43 of anti-envoplakin antibodies and BO were significantly associated with death; ii/ toxic 44 epidermal necrolysis-like clinical pattern, bullous pemphigoid-like clinical pattern and BO 45 were significantly associated with decreased survival.

$46 \quad$ Limitation Only case reports with sufficient mortality data were included.

47 Conclusion PNP associated with HM has a high mortality rate. TEN-like and BO-associated 48 forms are independent survival factors in PNP associated with HM. 


\section{Introduction}

54 Paraneoplastic pemphigus (PNP) is an autoimmune blistering skin disease associated with cancer that has various clinical presentations: lichen planus-like, pemphigus vulgaris-like, bullous pemphigoid (BP)-like, erythema multiforme-like, toxic epidermal necrolysis (TEN)like, chronic graft versus host disease-like (1). Skin or mucosal biopsies of PNP usually show a lichenoid pattern and/or patterns seen in autoimmune blistering skin diseases such as acantholysis, basal cells vacuolar degeneration, keratinocyte necrosis, sub-epidermal and/or epidermal cleft (2). PNP mechanism involves humoral and cellular autoimmunity (3) including an "epitope spreading" phenomenon with multiple antigen targets in the epidermis and the basal layer associated with various clinical presentations (4). After its first description in 1990 by Anhalt et al, diagnostic criteria to assess PNP were defined (2). They include the presence of a painful, progressive stomatitis and a polymorphous cutaneous eruption, histologic features of acantholysis, lichenoid or interface dermatitis, direct immunofluorescence demonstrating deposition of $\mathrm{IgG}$ and complement in the epidermal intercellular spaces, serum autoantibodies that bind the cell surface of skin and mucosae in a pattern typical for pemphigus, serum autoantibodies against desmogleins 1 and 3, as well as members of the plakin family of epithelial proteins. PNP occurs more often in patients with hematological malignancies (HM), especially B-cell lymphoproliferative disorders (LD), than in solid cancer patients (1)(5). Lung bronchiolitis obliterans (BO) is a usual complication of PNP, and can be lethal. PNP has a high mortality rate (6)(7). We aimed to accurately define PNP associated with HM and identify possible clinical and biological factors associated with mortality through a systematic review of previously described cases. 


\section{$75 \quad$ Material and Methods}

76 We searched the National Library of Medicine's MEDLINE database (Bethesda, MD) for

77 relevant case reports in the English and French literature using the keyword "Paraneoplastic

78 pemphigus" (1990 to 2016). The following criteria were used to select the cases: PNP meeting

79 the Anhalt criteria (2), HM associated with PNP, adult patients, biopsy proven HM, clinical,

80 histological and immunological characterization of the PNP and available data on mortality

81 and cause of mortality. Data were presented as median (interquartile range) or number

82 (percentage). Analysis of mortality rates first used logistic regression models. In patients with

83 available follow-up or survival times, Kaplan-Meier estimates of survival from PNP diagnosis

84 were plotted then compared across baseline groups by the log-rank test. Multivariable logistic

85 or Cox proportional hazards regression models including covariates with p-values $<0.05$ from

86 univariable analyses, were used to summarize prognostic information on mortality or survival,

87 respectively, both with model selection based on a backward stepwise selection procedure.

88 Strength of association between covariates and the outcome were measured by odds ratio

89 (OR) or hazard ratio (HRs) with 95\% confidence intervals (95\%CI).

90 Significance was defined by two-sided p-values less than 0.05. All statistical analyses were

91 performed using SAS software (SAS Institute Inc, Cary, NC), R (https://www.R-project.org/), 92 and GraphPad Prism Version 5.0 software (GraphPad Software). 


\section{Results}

\section{Patient selection and general characteristics}

One hundred and forty four patients were included for mortality rates including 120 who could be analyzed for survival data (see Flow chart in Figure 1). Patient characteristics are summarized in Table 1 to 7. Mean age was 58 years (48-68) with $44.4 \%$ of female patients. Clinical patterns were available for 76 patients (Table 2), accurate histological parameters for 137 patients (Table 3), direct immunofluorescence for 129 patients (Table 4), indirect immunofluorescence for 112 patients (Table 5), antigen targets (enzyme-linked immunosorbent assay or immunoblotting) for 112 patients (Table 6) and disease evolution criteria for 144 patients (Table 7). HM were non-Hodgkin lymphoma $(n=76,52.8 \%)$, chronic lymphocytic leukemia ( $n=33,22.9 \%)$, Castleman disease $(n=26,18.1 \%)($ unicentric, $n=24$; multicentric, $n=2)$, mastocytosis $(n=1,0.7 \%)$, Hodgkin Lymphoma $(n=1,0.7 \%)$ monoclonal gammopathy of undetermined significance/multiple myeloma/Waldenstrom ( $\mathrm{n}=7,4.9 \%)$.

Oral involvement was present in the majority of patients $(n=133,92.4 \%)$. Acantholysis was the most frequent histological pattern described $(n=86,62.7 \%)$, and sub epidermal blister was less frequent ( $\mathrm{n}=13,9.5 \%)$. Envoplakin (EVPL), periplakin (PPL) and desmoplakin were the most frequent antibody-targeted antigens $(n=81,72.3 \%, n=76,67.9 \%$ and $n=49 ; 43.7 \%$, respectively). BO was present in 37 patients (25.7\%). Relapse of HM was reported for 29 patients $(20.1 \%)$, relapse of PNP for 30 patients (20.8\%).

\section{Factors associated with mortality and survival time}

Eighty-two patients (57\%) died. The main cause of death was "general" for 37 patients (sepsis, general condition alteration), BO for 28 patients, PNP without BO (extended skin or mucosal involvement) for 16 patients and HM for 11 patients. Factors associated with increased mortality using univariate analysis were: absence of intra-epidermal blister, presence of anti-EVPL antibodies, presence of anti- PPL antibodies and presence of BO. The 
120 following criteria remained significantly associated with death using multivariate logistic

121 model: presence of anti-EVPL antibodies (OR=5.6, 95\%CI: 1.2-25.8; $\mathrm{p}=0.027)$ and $\mathrm{BO}$

$122(\mathrm{OR}=5.4,95 \% \mathrm{CI}: 1.7-16.9, \mathrm{p}=0.0034)$.

123 One-year survival was $62.4 \%$ (95\%CI: 53.8-72.4). Survival outcome is depicted in

124 Figure 2A. Factors associated with decreased survival in univariate analysis were: BP-like

125 pattern (Figure 2B), TEN-like pattern (Figure 2C), BO (Figure 2D) and anti-EVPL (Figure

126 2E). The following criteria remained significantly associated with survival using multivariate

127 Cox model: TEN-like pattern $(\mathrm{HR}=5.31,95 \% \mathrm{CI}: 2.6$ to $10.8, \mathrm{p}<0.001)$, BP-like pattern

$128(\mathrm{HR}=6.1,95 \% \mathrm{CI}: 1.30$ to $28.6, \mathrm{p}=0.022)$, and presence of $\mathrm{BO}(\mathrm{HR}=2.12,95 \% \mathrm{CI}: 1.14$ to

$1293.93, \mathrm{p}=0.018)$.

130

131

132 


\section{Discussion}

134 Our study is the first review from 1990 (first description of PNP) to 2016 concerning PNP 135 associated with HM that analyzed prognostic factors using either the prevalence of death or 136 the survival time as the endpoint. A French retrospective study of 50 patients analyzed HM

137 and solid neoplasms (carcinoma and sarcoma) and defined erythema multiform-like clinical 138 pattern and skin necrosis on histology (possibly seen in TEN-like PNP) as possible poor 139 prognostic factors (6). The four following factors were independently associated with 140 mortality and/or decreased survival in our study, namely presence of anti-EVPL antibodies, 141 BO, TEN-like clinical pattern, and BP-like clinical pattern. In mouse studies, EPVL appeared 142 to be a crucial protein for skin integrity and survival (8). BO has already been described as a 143 poor prognostic factor in PNP (9) (10). TEN-like PNP are often severe (11) but TEN-like 144 clinical pattern has not been already studied as an independent survival factor. BP-like clinical 145 pattern was associated with death in our study. However, only 3 patients had BP-like PNP 146 (the 3 of them died rapidly) making it difficult to draw firm conclusions. To summarize, the 147 survival rate of PNP associated with HM is low. TEN-like PNP and PNP associated with BO 148 are particularly severe. 


\section{Abbreviations}

154 PNP, paraneoplastic pemphigus; BP, bullous pemphigoid; HM, haematological malignancies;

155 TEN, toxic epidermal necrolysis; AVPL, envoplakin; PPL, periplakin 


\section{References}

1. Wieczorek M, Czernik A. Paraneoplastic pemphigus: a short review. Clin Cosmet Investig Dermatol. 2016;9:291-5.

2. Anhalt GJ, Kim S, Stanley JR, Korman NJ, Jabs DA, Kory M, et al. Paraneoplastic Pemphigus: An Autoimmune Mucocutaneous Disease Associated with Neoplasia. N Engl J Med. 1990 Dec 20;323(25):1729-35.

3. Huang Y, Li J, Zhu X. Detection of anti-envoplakin and anti-periplakin autoantibodies by ELISA in patients with paraneoplastic pemphigus. Arch Dermatol Res. 2009 Oct;301(10):703-9.

4. Kartan S, Shi VY, Clark AK, Chan LS. Paraneoplastic Pemphigus and Autoimmune Blistering Diseases Associated with Neoplasm: Characteristics, Diagnosis, Associated Neoplasms, Proposed Pathogenesis, Treatment. Am J Clin Dermatol. 2017 Feb $1 ; 18(1): 105-26$.

5. Kaplan I. Neoplasms associated with paraneoplastic pemphigus: a review with emphasis on non-hematologic malignancy and oral mucosal manifestations. Oral Oncol. 2004 Jul;40(6):553-62.

6. Leger S, Picard D, Ingen-Housz-Oro S, Arnault J-P, Aubin F, Carsuzaa F, et al. Prognostic Factors of Paraneoplastic Pemphigus. Arch Dermatol. 2012 Oct $1 ; 148(10): 1165$.

7. The Mechanism of Respiratory Failure in Paraneoplastic Pemphigus. N Engl J Med. 1999 Sep 9;341(11):848-848.

8. Sevilla LM, Nachat R, Groot KR, Klement JF, Uitto J, Djian P, et al. Mice deficient in involucrin, envoplakin, and periplakin have a defective epidermal barrier. J Cell Biol. 2007 Dec 31;179(7):1599-612.

9. Zhen J-F, Zhang L, Cao X-X, Feng J, Zhou D-B, Lin S-B, et al. Clinical Analysis of Unicentric Castleman's Disease with Paraneoplastic Pemphigus and Bronchiolitis Obliterans. Zhongguo Yi Xue Ke Xue Yuan Xue Bao. 2017 20;39(4):492-8.

10. Ohzono A, Sogame R, Li X, Teye K, Tsuchisaka A, Numata S, et al. Clinical and immunological findings in 104 cases of paraneoplastic pemphigus. Br J Dermatol. 2015 Dec;173(6):1447-52.

11. McLarney RM, Valdes-Rodriguez RH, Isaza-Gonzalez G, Miller JH, Hsu S, Motaparthi K. Paraneoplastic pemphigus mimicking toxic epidermal necrolysis: An underdiagnosed entity? JAAD Case Rep. 2017 Dec 19;4(1):67-71. 


\section{Figure Legends}

194 Figure 1: Flow chart of patient selection

195 Figure 2: A. Kaplan Meier probabilities of overall survival in 120 patients with

196 paraneoplastic pemphigus. B. Probabilities of overall survival in patients with paraneoplastic

197 pemphigus, according to the presence or absence of a bullous-pemphogoid like pattern. C.

198 Probabilities of overall survival in patients with paraneoplastic pemphigus, according to the

199 presence or absence of a toxic epidermal necrosis-like pattern. D. Probabilities of overall

200 survival in patients with paraneoplastic pemphigus, according to the presence or absence of

201 bronchiolitis obliterans. E. Probabilities of overall survival in patients with paraneoplastic

202 pemphigus, according to the presence or absence of anti-envoplakine antibodies. p-values are

203 estimated using the log-rank test 
206 Table 1: General characteristics of the 144 patients

\begin{tabular}{llll}
\hline & Number or Mean & Percentage or interquartile \\
\hline Age & & 58 & $48-68$ \\
Sex & F & & \\
& M & 64 & $44.4 \%$ \\
Hematologic malignancies & 80 & $55.6 \%$ \\
CLL & 33 & $22.9 \%$ \\
NHL & 76 & $52.8 \%$ \\
HL & 1 & $0.7 \%$ \\
CD & 26 & $18.1 \%$ \\
Mastocytosis & 1 & $0.7 \%$ \\
Monoclonal gammopathy & 7 & $4.9 \%$ \\
\hline \hline
\end{tabular}

F: Female; M: Male; CLL: chronic lymphocytic leukemia; NHL: non Hodgkin lymphoma; HL: Hodgkin lymphoma CD: Castleman's disease 
211 Table 2: Clinical characteristics of the 144 patients

212

\begin{tabular}{ll}
\hline Settings & Number (percentage for 144 patients) \\
\hline Skin involvement & $128(88.9 \%)$ \\
Lichen planus-like & $37(25.7 \%)$ \\
GVH-like & $4(2.8 \%)$ \\
BP-like & $3(2.1 \%)$ \\
VP-like & $15(10.4 \%)$ \\
Erythema multiform like & $17(11.8 \%)$ \\
TEN-like & $17(11.8 \%)$ \\
No pattern described & $68(47.2 \%)$ \\
Oral involvement & $133(92.4 \%)$ \\
Genital involvement & $47(32.6 \%)$ \\
Anal involvement & $12(8.3 \%)$ \\
Eye involvement & $72(50 \%)$ \\
ENT involvement & $28(19.4 \%)$ \\
Extremities & $71(49.3 \%)$ \\
Blisters & $91(63.6 \%)$ \\
\hline
\end{tabular}

GVH: graft versus host disease BP: bullous pemphigoid; VP: vulgaris pemphigus; TEN: toxic epidermal necrolysis; ENT: ears nose throat pharynges or epiglottis involvement

Note: Two and sometimes three clinical patterns could be described for one patient 
220 Table 3: Histological characteristics (137 patients with available data)

221

\begin{tabular}{ll}
\hline & Number (percentage) \\
\hline Intra-epidermal blister & $59(43.1 \%)$ \\
Sub-epidermal blister & $13(9.5 \%)$, \\
Acantholysis & $86(62.7 \%)$ \\
Keratinocyte necrosis & $69(50.4 \%)$ \\
Basal cells degeneration & $45(32.8 \%)$ \\
Lichenoid infiltrate & $74(54.0 \%)$ \\
\hline \hline
\end{tabular}

222

223

Note histological pattern are often multiple in one patient which is common in PNP

224 
225 Table 4: Skin biopsy direct immunofluorescence (129 patients with available data)

226

\begin{tabular}{ll}
\hline & Number (percentage) \\
\hline IgG & $108(83.7 \%)$ \\
$\mathrm{C} 3$ & $92(71.3 \%)$ \\
$\mathrm{C} 1 \mathrm{q}$ & $5(3.8 \%)$ \\
$\mathrm{IgM}$ & $11(8.5 \%)$ \\
IgA & $9(7.0 \%)$ \\
Intercellular only & $41(31.8 \%)$ \\
Linear only & $6(4.6 \%)$ \\
Linear and intercellular & $71(55.0 \%)$ \\
negative DIF & $11(8.5 \%)$
\end{tabular}

IgG: G-type immunoglobulin deposit; C3: Fraction number 3 of complement deposits; C1q: component $q$ of the fraction number 1 of complement deposit; IgM: M-type immunoglobulin deposit; IgA: A-type immunoglobulin deposit; Intercellular: deposits are localized between keratinocyte in the epidermis following desmosome formation; Linear: deposits are localized along basal membrane following hemi desmosome, lamina densa or lamina lucida; DIF: direct immunofluorescence analysis 
234 Table 5: Indirect Immunofluorescence (112 patients with available data)

\begin{tabular}{ll}
\hline Settings & Number (percentage) \\
\hline Rat bladder & $94(83.9 \%)$ \\
ICS only & $66(58.9 \%)$ \\
BMZ only & $4(3.6 \%)$ \\
ICS and BMZ & $12(10.7 \%)$ \\
IIF negative & $8(7.1 \%)$ \\
\hline \hline
\end{tabular}

IIF: indirect immunofluorescence; ICS: intercellular substance antibody; BMZ: basal membrane zone antibody. Depending on the case reported, substrates for Indirect Fluorescence were mostly monkey oesophagus, monkey bladder, rat bladder and sometimes a combination of these. 
241

242 Table 6: ELISA and Immunoblot (data available for 112 patients)

\begin{tabular}{ll}
\hline Settings & Number (percentage over 112 patients) \\
\hline BP 180 (180 kD) & $8(7.1 \%)$ \\
BP 230 (230 kD) & $38(33.9, \%)$ \\
Dsg1 (160kD) & $21(18.7 \%)$ \\
Dsg3 (130 kD) & $45(40.2 \%)$ \\
Alpha 2 macroglobulin like 1 (170kD) & $22(19.6 \%)$ \\
Periplakin (190 kD) & $76(67.9 \%)$ \\
Laminin 3-3-2 & $2(1.8 \%)$ \\
Collagen VII alpha chain (290kD) & $2(1.8 \%)$ \\
Desmocollin & $5(4.4 \%)$ \\
Envoplakin $(210 \mathrm{kD})$ & $81(72.3 \%)$ \\
Desmoplakin $(250 \mathrm{kD})$ & $49(43.7 \%)$
\end{tabular}

BP, Bullous Pemphigoid; Dsg, Desmoglein 
247 Table 7: Evolution and outcome of the 144 patients

248

Number (percentage)

\begin{tabular}{ll}
\hline BO & $37(25.7 \%)$ \\
Relapse PNP & $30(20.8 \%)$ \\
Relapse HM & $29(20.1 \%)$ \\
Deaths & $82(56.9 \%)$ \\
Death consecutive to BO & $28 / 82(34.1 \%)$ \\
Death consecutive to PNP & $16 / 82(19.5 \%)$ \\
Death consecutive to HM & $11 / 82(13.4 \%)$ \\
Death consecutive to other causes & $37 / 82(45.1 \%)$
\end{tabular}

BO: bronchiolitis obliterans; PNP: paraneoplastic pemphigus; HM, hematologic malignancy; Relapse PNP: relapse of paraneoplastic pemphigus during patient follow up; Relapse HM: relapse of hematologic malignancy during patient follow up; Other causes: other causes than bronchiolitis obliterans s, hematological malignancy and paraneoplastic pemphigus 
Criteria not met

Paediatric cases: 22

Solid neoplasia: 74

Veterinary studies: 4

No neoplasia related to PNP: 10

Review without case report: 7

No details on the reported cases: 53

Anhalt criteria not met: 19

Other language than french or english: 11

120 patients for survival analysis 


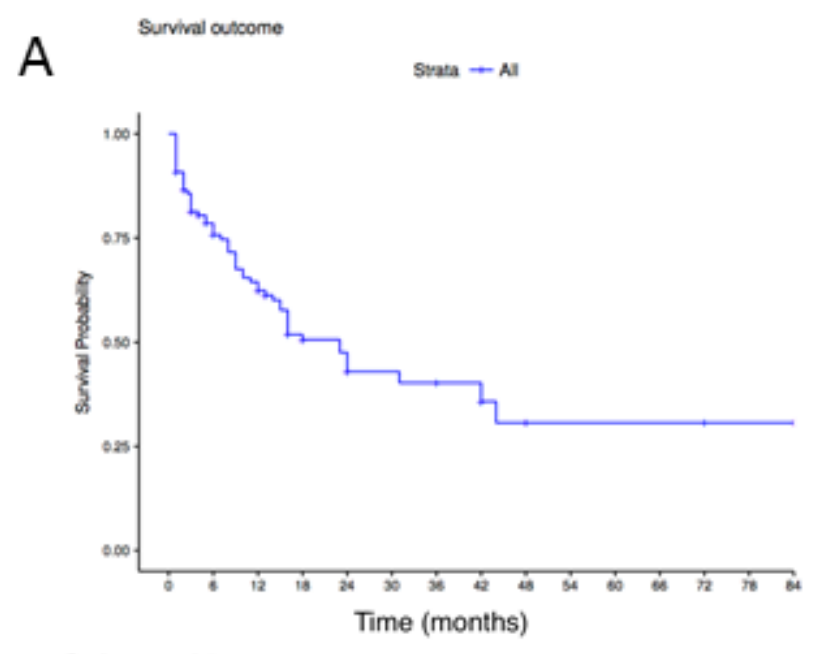

Patients at risk

All 120 10 $60 \begin{array}{lllll}4 & 39 & 16 & 15 & 9\end{array}$

B

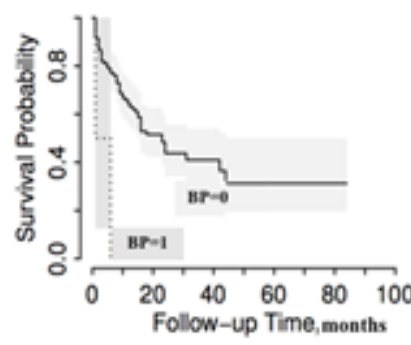

$P=0.009$
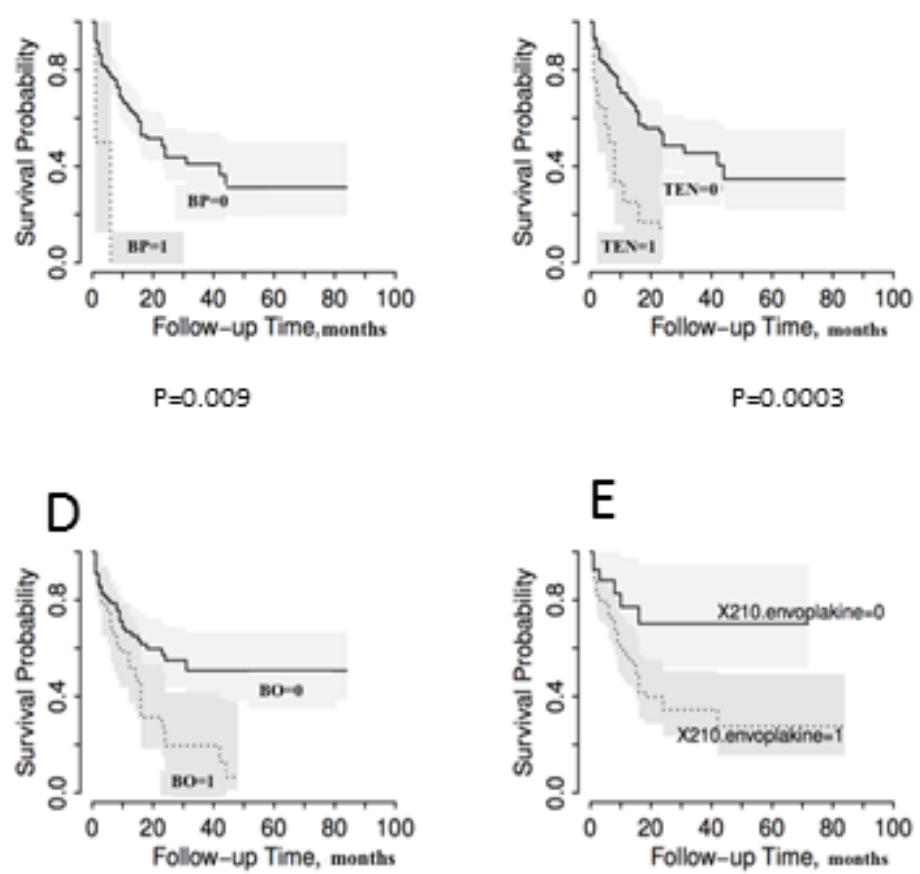

$P=0.0003$

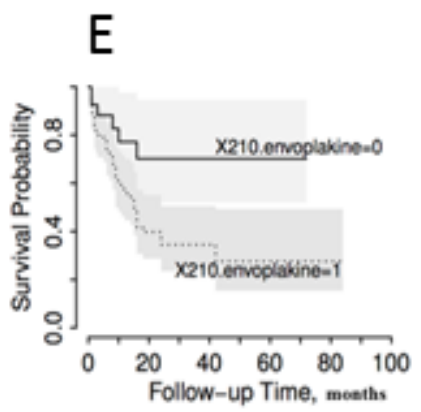

